\title{
PROCESSAMENTO DE DOCE EM PASTA DO ARATICUM (Annona crassiflora Mart.) E VIABILIDADE DA INSERÇÃO NA ALIMENTAÇÃO ESCOLAR
}

\author{
Araticum (Annona crassiflora Mart.) Paste Sweet Processing And Insertion Feasibility \\ in School
}

Araticum (Annona crassiflora Mart.) Pasta Dulce Procesamiento E Inserción Factibilidad en la Escuela

\section{Maria Olivia dos Santos Oliveira*2, Bianca Barros Dias², Rômulo Alves Morais ${ }^{1,2}$, Glêndara Aparecida de Souza Martins ${ }^{1,2}$}

${ }^{1}$ Programa de Pós-graduação em Ciência e Tecnologia de Alimentos, Universidade Federal do Tocantins, PalmasTocantins, Brasil.

${ }^{2}$ Laboratório de Cinética e Modelagem de Processos, Universidade Federal do Tocantins, Palmas-Tocantins, Brasil.

*Correspondência: Laboratório de Cinética e Modelagem de Processos, Av. NS 15, 109 Norte, Palmas, Tocantins, Brasil.CEP:77.010-090.e-mail mariaoliviaeng@gmail.com

\section{Artigo recebido em 11/02/2020 aprovado em 27/03/2020 publicado em 31/03/2020.}

\section{RESUMO}

Araticum (Annona crassiflora Mart.) é um fruto nativo do bioma Cerrado que possui alto potencial nutricional, funcional e econômico. Os frutos do cerrado são alvo constante de pesquisas e estudos que comprovam sua importância cultural, nutricional e alimentar, podendo ser consumidos in natura ou processados na forma de doces, geleias, entre outros. O processamento desses frutos, além de agregar valor, permite o aumento da vida útil e o consumo de produtos independente do período de colheita. Portanto, o presente trabalho tem como objetivo o aproveitamento do fruto do araticum, na forma de doce em pasta com substituição da pectina comercial por albedo de maracujá, rotulagem nutricional e avaliação sensorial de crianças da rede pública de Palmas-TO, com foco na aplicação desse produto na alimentação escolar. O doce foi produzido com 50/50 partes de polpa/açúcar, 1,5\% de albedo do maracujá e $0,5 \%$ de ácido cítrico, então foi procedida a rotulagem nutricional e sensorial com 50 crianças em uma escola da rede pública em Palmas-TO. O doce apresentou baixo valor calórico, ficando abaixo do limite estipulado pelo FNDE para doces na alimentação escolar, ainda apresentou alto valor nutricional e foi bem aceito pelas crianças.

Palavras-chave: frutos do cerrado, alimentação escolar, doce.

\section{ABSTRACT}

Araticum (Annona crassiflora Mart.) Is a native fruit of the Cerrado biome that has high nutritional, functional and economic potential. The fruits of the Cerrado are a constant target of research and studies that prove their cultural, nutritional and food importance, and can be consumed fresh or processed as sweets, jellies, among others. The processing of these fruits, besides adding value, allows the increase of shelf life and the consumption of products regardless of the harvesting period. Therefore, the present work aims to use the fruit of araticum, in the form of sweet paste with substitution of commercial pectin for passion fruit albedo, nutritional labeling and sensory evaluation of children from the public school of Palmas-TO, focusing on the application. this product in school meals. The candy was made with 50/50 parts of pulp / sugar, $1.5 \%$ passion fruit albedo and $0.5 \%$ citric acid, so nutrition and sensory labeling were performed with 50 children in a public school in Palmas -TO.

Keywords: cerrado fruits, school meals, sweet. 


\section{RESUMEN}

Araticum (Annona crassiflora Mart.) Es una fruta nativa del bioma Cerrado que tiene un alto potencial nutricional, funcional y económico. Los frutos del Cerrado son un objetivo constante de investigación y estudios que demuestran su importancia cultural, nutricional y alimentaria, y se pueden consumir frescos o procesados como dulces, jaleas, entre otros. El procesamiento de estas frutas, además de agregar valor, permite el aumento de la vida útil y el consumo de productos independientemente del período de cosecha. Por lo tanto, el presente trabajo tiene como objetivo utilizar la fruta de araticum, en forma de pasta dulce con sustitución de pectina comercial por albedo de maracuyá, etiquetado nutricional y evaluación sensorial de niños de la escuela pública de Palmas-TO, centrándose en la aplicación. Este producto en las comidas escolares. El dulce se hizo con 50/50 partes de pulpa / azúcar, 1.5\% de albedo de maracuyá y $0.5 \%$ de ácido cítrico, por lo que se realizó nutrición y etiquetado sensorial con 50 niños en una escuela pública en Palmas -TO. Los dulces tenían un bajo valor calórico, estaban por debajo del límite establecido por la FNDE para los dulces en las comidas escolares, todavía tenían un alto valor nutricional y eran bien aceptados por los niños.

Descriptores: frutas cerradas, comidas escolares, dulces.

\section{INTRODUÇÃO}

O cerrado é o segundo maior bioma do Brasil, ocupa $22 \%$ do território nacional, sendo superado apenas pela Amazônia (BRASIL, 2017). Devido a sua extensão e situação geográfica, a região é caracterizada por grandes variações de solo, clima, fauna e flora. O clima é sazonal, ou seja, composto por um período chuvoso que se estende de outubro a março e pelo período seco que abrange os meses de abril a setembro. (KLINK; MACHADO, 2005; MALTA 2011; CHUNG, 2016).

A flora do cerrado brasileiro possui grande diversidade de frutos com grande potencial agrícola. Pelo fato dessa grande diversidade frutífera, pouco conhecimento das características e dos benefícios que o consumo in natura ou processados podem proporcionar à saúde humana, o estudo sobre os frutos do cerrado tem se tornado frequentes dentro da região (CHUNG, 2016). Assim, além do consumo in natura, essas frutas podem ser transformadas em sucos, sorvetes, doces, licores, pães e bolos provocando agregação de valor a matéria prima e o aumento de sua vida útil. (MALTA, 2011; ANGELLA, 2014). Dentre os processos utilizados para agregação de valor a frutos, a elaboração de doces é considerada uma das formas mais aceitas, uma vez que promove a melhoria da qualidade dos produtos ofertados e ampliação da sua vida útil. (FREITAS et al, 2012).

Nesse contexto o araticum é um fruto nativo do cerrado cujo a polpa é rosada ou amarelada, adocicada e rica em carotenoides, polifenois, tocoferóis, flavonoides, vitaminas e minerais (AGUIAR; SOUZA; OLIVEIRA, 2018). Seu consumo ocorre prioritariamente na forma in natura, no entanto, seu processamento é desejável face a sua frutificação exclusiva a partir dos meses de janeiro e fevereiro, o que dificulta o seu consumo.

Não obstante, destaca-se que a alimentação desempenha um papel importantíssimo durante todo o ciclo de vida de um indivíduo, mas nos primeiros anos de vida esta deve ser qualitativamente e quantitativamente adequada, com o propósito de garantir a ingestão de nutrientes necessários para o crescimento e desenvolvimento (LLOYD-WILLIAM et al., 2010; JENNINGS et al., 2012). Neste ciclo destaca-se a fase escolar, que se caracteriza por um período em que a criança apresenta um metabolismo muito mais intenso quando comparado ao do adulto e são desenvolvidos hábitos alimentares. Com isso, torna-se essencial que alimentos com propriedades nutricionais importantes sejam oferecidos às crianças tanto no domicílio como na escola (BRASIL, 2013; HERMAN et al., 2014; INOUE et al., 2015). 
Os critérios estabelecidos pela legislação vigente para uma alimentação correta em creches e escolas podem sofrer a influência de fatores intrínsecos e extrínsecos, tais como problemas no fornecimento de matérias-primas, dificuldades na substituição de itens dentro de um grupo alimentar e consequente não atendimento das quantidades energéticas necessárias, podendo desencadear doenças de origem alimentar. Diante disso, o uso de matériasprimas nativas facilita o acesso a fontes nutricionais importantes e minimiza o impacto de problemas logísticos. Os frutos do cerrado são alvo constante de pesquisas e estudos que comprovam sua importância cultural, nutricional e alimentar. $\mathrm{O}$ processamento desses frutos, além de agregar valor, permite o aumento da vida útil e o consumo de produtos independente do período de colheita.

Diante do exposto, este trabalho tem como objetivo o aproveitamento do fruto do araticum, na forma de doce em pasta com substituição da pectina comercial por albedo de maracujá, rotulagem nutricional e avaliação sensorial de crianças da rede pública de Palmas-TO, com foco na aplicação desse produto na alimentação escolar.

\section{MATERIAIS E MÉTODOS}

A pesquisa foi submetida previamente ao Comitê de Ética em Pesquisa da Universidade Federal do Tocantins, o qual foi aprovado com o Certificado de Apresentação para Apreciação Ética número 93357718.3.0000.5519 e parecer número 3.096.189.

As polpas integrais do fruto para preparo dos doces foram disponibilizadas pela cooperativa Grande Sertão, de Montes Claros - MG, encaminhadas para o Laboratório de Cinética e Modelagem de Processos na Universidade Federal do Tocantins.

A formulação para o preparo dos doces foi selecionada através de testes anteriores, na qual apresentou proporção de 50 partes de açúcar e 50 partes de polpa, 1,5\% de albedo de maracujá e 0,5\% de ácido cítrico. Para o preparo foram seguidas as etapas: formulação, composta por açúcar, polpa e albedo, então foi concentrado à pressão atmosférica, adicionando ácido cítrico no final do processo, esse procedimento deve ser realizado nessas condições já que a pectina sob aquecimento em meio ácido sofre hidrólise, com perda do seu poder geleificante (SOLER et al., 1995). O processamento foi sessado quando o doce chegou a $78{ }^{\circ}$ Brix. Por fim, foi realizado enchimento à quente e fechamento da embalagem. Utilizou-se o albedo de maracujá como fonte de pectina, conforme descrito por Silva et al., (2012).

\section{Rotulagem Nutricional}

A tabela de informação nutricional do rótulo do doce foi elaborada através de cálculos na ferramenta Excel, atendendo a legislação vigente da ANVISA. a RDC 359 que trata do Regulamento Técnico de Porções de Alimentos Embalados Para Fins de Rotulagem Nutricional e a RDC 360 que aborda o Regulamento Técnico Sobre Rotulagem Nutricional de Alimentos Embalados, incorporando as normas aprovadas no MERCOSUL (BRASIL, 2003a; Brasil, 2003b).

\section{Análise Sensorial com as crianças}

Os testes foram realizados em uma escola pública localizada em Palmas-TO. Onde foram servidos as crianças já alfabetizadas amostras de $15 \mathrm{~g}$ do doce em copos de plástico de 50mL, acompanhados de água e bolachas de água e sal (STONE E SIDEL, 2005). Durante a degustação deles foi explicado sobre o fruto e como o doce foi produzido.

No intuito de avaliar a aceitação por parte de crianças e possível viabilidade para inserção dos produtos na alimentação escolar foi realizada a aplicação de teste afetivo a crianças já alfabetizadas, 
com idades entre 8 e 12 anos, com escala hedônica de expressão facial com cinco categorias similar a proposta por Gastaldon et al. (2007) (Figura 1). Esse método utiliza como forma de avaliação desenhos expressando o quanto gostou ou desgostou da amostra e é recomendado para análise de produtos destinados ao consumo infantil (HURSTI, 1999).

Figura 1. Modelo de escala hedônica apresentada a crianças.

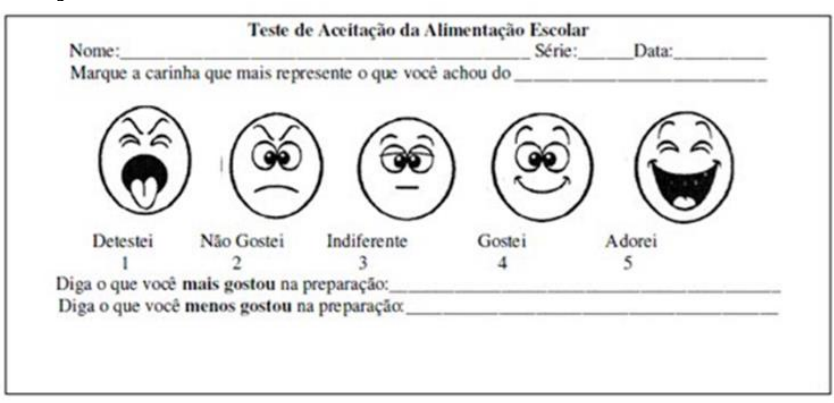

\section{RESULTADOS E DISCUSSÃO}

A rotulagem nutricional é toda a descrição destinada a informar o consumidor sobre as propriedades nutricionais de um alimento, compreendendo a declaração de valor energético e os principais nutrientes. Garantindo que estas informações sejam compreendidas por todos aqueles que as utilizam (ANVISA e UnB, 2005; SOUZA et al., 2011).

As informações fornecidas por meio da rotulagem contemplam um direito assegurado pelo Código de Defesa do Consumidor, que determina que a informação sobre produtos deve ser clara e com especificação correta de quantidade, composição e qualidade, bem como sobre os riscos que possam apresentar ao consumidor (BRASIL, 1990; CÂMARA et al., 2008). A rotulagem dos alimentos, ao orientar o consumidor sobre a qualidade e a quantidade dos constituintes nutricionais dos produtos, pode promover escolhas alimentares apropriadas. Encontrase na tabela 1 a composição nutricional da formulação.
Tabela 1. Valores de composição nutricional da melhor formulação do doce em pasta.

INFORMAÇÃO NUTRICIONAL Porção de 20 g (1 colher de sopa)

\begin{tabular}{c|c|c}
\hline & $\begin{array}{c}\text { Quantidade por } \\
\text { porção }\end{array}$ & $\begin{array}{c}\text { \% VD } \\
(*)\end{array}$ \\
\hline Valor Energético & $\mathbf{8 0 ~ K c a l = 3 3 4 ~ K J ~}$ & $\mathbf{4}$ \\
\hline Carboidratos & $\mathbf{1 9} \mathbf{~ g}$ & $\mathbf{6}$ \\
\hline Proteínas & $\mathbf{0 . 2} \mathbf{g}$ & $\mathbf{0 . 3}$ \\
\hline Gorduras Totais & $\mathbf{0 . 3} \mathbf{g}$ & $\mathbf{0 . 6}$ \\
\hline Fibra Alimentar & $\mathbf{0 . 4} \mathbf{g}$ & $\mathbf{2}$ \\
\hline Sódio & $\mathbf{0 . 7} \mathbf{~ m g}$ & $\mathbf{0}$ \\
\hline Cálcio & $\mathbf{3 . 3} \mathbf{~ m g}$ & $\mathbf{0 . 3}$ \\
\hline Fosforo & $\mathbf{6 . 7} \mathbf{~ m g}$ & $\mathbf{1}$ \\
\hline Vitamina A & $\mathbf{8 . 3} \mathbf{~ m g ~ R E}$ & $\mathbf{1 . 4}$ \\
\hline Vitamina C & $\mathbf{9 . 5} \mathbf{~ m g}$ & $\mathbf{2 1 . 1}$
\end{tabular}

(*)\% Valores Diários de referência com base em uma dieta de $2.000 \mathrm{kcal}$ ou $8400 \mathrm{~kJ}$. Seus valores diários podem ser maiores ou menores dependendo de suas necessidades energéticas.

O cardápio da alimentação escolar é um instrumento de planejamento que visa assegurar a oferta de uma alimentação saudável e adequada, que garanta $\mathrm{o}$ atendimento das necessidades nutricionais dos alunos durante o período letivo.

No entanto, a oferta da alimentação escolar configura um elemento pedagógico, caracterizando uma importante ação de educação alimentar e nutricional. O PNAE, visando limitar a oferta de alimentos processados de baixo valor nutricional, estabeleceu em sua normativa, Resolução CD/FNDE $\mathrm{n}^{0} 26$ de 17 de junho de 2013, a oferta de doces e/ou preparações doces fica limitada a duas porções por semana, equivalente a $110 \mathrm{kcal} /$ porção.

Portanto, de acordo com o contexto apresentado a inserção do Araticum na alimentação escolar seria uma alternativa viável, devido ao seu valor nutricional e a porção equivalente a $80 \mathrm{kcal}$. Sua disposição durante apenas um período do ano, não implicaria em sua disponibilidade, devido a possibilidade de processamento, podendo ser inserido na alimentação de crianças e jovens sob a forma de alimentos que consomem rotineiramente.

O teste de aceitação realizado com as crianças foi realizado com a formulação otimizada, ou seja, a 
que apresentou melhores condições no rendimento e aceitação sensorial de adultos.

Participaram dos testes de aceitação sensorial do doce de araticum 50 provadores não treinados onde $60 \%$ (30) eram do sexo masculino e $40 \%$ (20) do sexo feminino, com idades entre 8 e 10 anos, todos estudantes da rede municipal do estado do Tocantins. O somatório das notas atribuídas ao doce está apresentados logo abaixo na figura 2 .

Figura 2. Histograma de escores de notas quanto a aceitação das crianças.

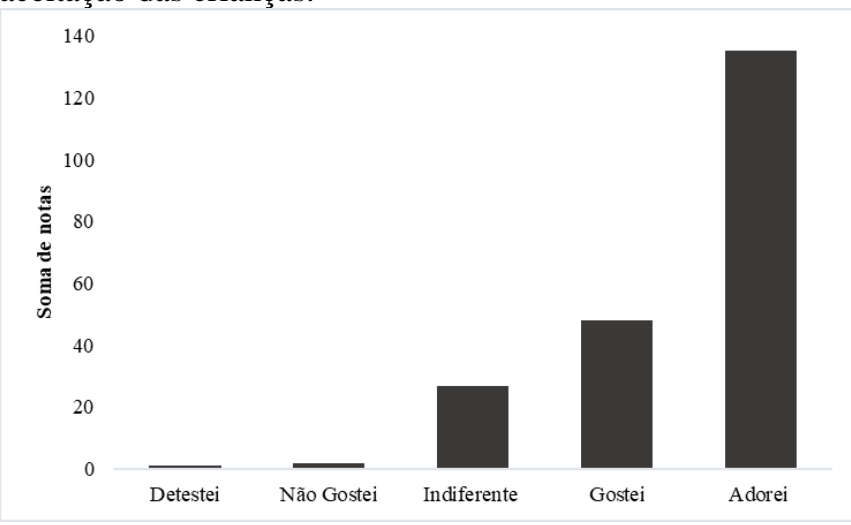

Carvalho et al. (2015) selecionaram 16 estudos, publicados entre 2003 e 2013, a respeito do consumo alimentar e adequação nutricional em crianças brasileiras. Notaram que a prevalência de inadequação de micronutrientes variou de $0,4 \%$ a $65 \%$ para o ferro, $20 \%$ a $59,5 \%$ para a vitamina A, $20 \%$ a $99,4 \%$ para o zinco, $12,6 \%$ a $48,9 \%$ para o cálcio e de $9,6 \%$ a $96,6 \%$ para a vitamina $\mathrm{C}$.

Já Vieira (2017) comprova em seu estudo que existem diferenças na qualidade nutricional de padrões alimentares dentro e fora das escolas. Além disso, foi observou heterogeneidade na adesão a esses padrões em todas as regiões e classes socioeconômicas. Este estudo reforça a importância de ações de educação nutricional e políticas públicas mais eficazes na promoção de escolhas alimentares mais saudáveis na infância.

Cardoso et al. (2013) expõem que o Araticum é uma importante alternativa alimentar que pode DOI: http://dx.doi.org/10.20873/uftsupl2020-8432 contribuir para o acesso a dietas, especialmente em regiões com alta níveis de insegurança alimentar e em locais onde pode haver escassez ou ausência de alimentos considerados como fontes desses nutrientes na dieta brasileira. Podendo funcionar como fontes alternativas de alimentos compondo cardápios regionalizados, podendo ser fornecidas até mesmo na merenda escolar.

\section{CONCLUSÃO}

O doce foi bem aceito pelas crianças, este já é o um grande passo para atestar a viabilidade da inserção na alimentação escolar. Vale ressaltar que deverão ser realizados testes de custos e cálculo de nutrientes diários e avaliação dos nutricionistas responsáveis pelos cardápios das escolas.

Os resultados mostraram um produto saboroso, rico nutricionalmente a partir de frutos nativos, utilizando resíduo na formulação e aplicando uma tecnologia para agregar valor e a disponibilidade do fruto durante o ano inteiro. Valorizando o bioma e incentivando a inserção e o consumo de frutos nativos na alimentação, não só no estado mais em todo o Brasil.

\section{AGRADECIMENTO}

A Universidade Federal do Tocantins, CNPq e CAPES.

Todos os autores declararam não haver qualquer potencial conflito de interesses referente a este artigo.

\section{REFERÊNCIAS}

AGÊNCIA NACIONAL DE VIGILÂNCIA SANITÁRIA - ANVISA; UNIVERSIDADE DE BRASÍLIA - UnB. Rotulagem Nutricional Obrigatória: Manual de Orientação às Indústrias de Alimentos. 2. versão. Brasília: ANVISA, UnB, 44 p. 2005.

AGUIAR, A. O. ; SOUZA, A. R. M. de; OLIVEIRA, M. O. dos S. Araticum (Annona Crassiflora Mart.) In: Frutos do Cerrado: características e aplicações Revista Desafios - Suplemento, 2020 
tecnológicas. I Adriana Régia Marques de Souza, Clarissa Daminani, Glêndara Aparecida de Souza Martins, Juliana Fonseca Moreira da Silva (organizadoras) - Curitiba: CRV, 148p. 2018.

ANGELLA, F . C. DE O. Avaliação da atividade antioxidante em extratos de frutas típicas do Cerrado brasileiro. 2014. 79 f. Tese (Doutorado) - Curso de Química Analítica e Inorgânica, Universidade de São Paulo, São Carlos, 2014.

BRASIL. Ministério da Justiça. Código de Defesa do Consumidor (CDC). Lei $\mathrm{n}^{\circ}$ 8.078/90, de 11 de setembro de 1990. Dispõe sobre a proteção do consumidor e dá outras providências. Diário Oficial da República Federativa do Brasil, Poder Executivo, Brasília, DF, 12 set. 1990. Seção 1.

BRASIL. AGÊNCIA NACIONAL DE VIGILÂNCIA SANITÁRIA (ANVISA). RDC 359 de 23 de setembro de 2003. 2003a. Dispõe sobre Regulamento Técnico de porções de alimentos embalados para fins de rotulagem nutricional. Brasília, 2003.

BRASIL. Resolução CD/FNDE $n^{\circ} 26$, de 17 de junho de 2013. Dispõe sobre o atendimento da alimentação escolar aos alunos da educação básica no âmbito do Programa Nacional de Alimentação Escolar- PNAE. Diário Oficial da União.2013.

BRASIL, Ministério do Meio Ambiente (MMA). O Bioma Cerrado. 2017. Disponível em: http://www.mma.gov.br/biomas/cerrado. Acesso em 10 de dezembro de 2018.

CÂMARA, M. C. C.; MARINHO, C. L. C.; GUILAM, M. C.; BRAGA, A. M. C. B. A produção acadêmica sobre a rotulagem de alimentos no Brasil. Revista Panamericana de Salud Pública, Washington, v. 23, n. 1, p. 52-58, 2008.

CARDOSO, L.; OLIVEIRA, D.; BEDETTI, S.; MARTINO, H.; PINHEIRO-SANT'ANA, H.; Araticum (Annona crassiflora Mart.) from the Brazilian Cerrado: Chemical composition and bioactive compounds. Fruits, 68(2), 121-134. 2013.

CARVALHO, C. A. de; FONSÊCA, P. C. de A.; PRIORE, S. E., FRANCESCHINI, S. D. C. C.; DE NOVAES, J. F. (2015). Consumo alimentar e adequação nutricional em crianças brasileiras: revisão sistemática. Revista Paulista de Pediatria, 33(2), 211-221.

CHUNG, MONIQUE MI SONG. Polpa de guavira (Campomanesia cambessedeana Berg) desidratada em spray dryer: efeitos das condições de processo e composição da alimentação nas propriedades físico químicas e atividade antioxidante. Tese de Doutorado. Universidade de São Paulo. 2016.
FREITAS, M. L. F.; MENEZES, C. C.; CARNEIRO, J. D. S.; REIS, R.P. Consumo e produção de doces artesanais. Alim. Nitr., Araraquara, v. 23, n. 4, p. $589-595,2012$.

GASTALDON, B.; MARTINS, J. C.; POLTRONIERI, K. V. Obesidade Infantil: um problema do presente com olhares para o futuro promovendo o ser e a família saudável no quotidiano junto à enfermagem. Florianópolis, 2007.

HERMAN, D.R.; BAER, M.T.; ADAMS, E.; CUNNINGHAAMSABO L.; DURAN, N.; JOHNSON, D. B.; et al. Life course perspective: Evidence for the role of nutrition. Maternal and child health jornal, v. 18, n. 2, p. 450-61. 2014.

HURSTI, Ulla-Kaisa Koivisto. Factors influencing children's food choice. Annals of medicine, v. 31, n. sup1, p. 26-32, 1999.

INOUE D. Y.; OSÓRIO, M. M.; TACONELI, C. A.; SCHMIDT, S. T.; ALMEIDA, C. C. B. Consumo alimentar de crianças de 12 a 30 meses que frequentam Centros Municipais de Educação Infantil no município de Colombo, Sul do Brasil. Rev. Nutr., Campinas, v. 28, n. 5, p. 523-532, 2015.

JENNINGS, A.; MCEVOY, S.; CORISH C. Nutritional practices in full-day-care pre-schools. J Hum Nutr Diet. v. 24, n. 3, p. 245-56. 2012.

KLINK, C.A.; MACHADO, R.B. A conservação do Cerrado brasileiro. Megadiversidade, v. 1, n. 1, p. 147 $-155,2005$.

LOYD-WILLIAM, F.; BRISTOW, K.; CAPEWELL, S.; MWATSAMA, M. Young children's food in Liverpool day-care settings: A qualitative study of preschool nutrition policy and practice. Pub Health Nutr. V. 14, n. 10 p. 1858-66. 2010.

MALTA, L.G. Avaliação biológica de frutas do cerrado brasileiro: guapeva, gabiroba e murici. 2011. 224 f. Tese (Doutorado) - Curso de Ciência de Alimentos, Faculdade de Engenharia de Alimentos, Unicamp, Campinas - SP, 2011.

SILVA, I.G.; MARTINS, G.A.S.; BORGES, S.V.; MARQUES, G.R; REGIS, I.S. Influence of passion fruit albedo, citric acid, and a the pulp/sugar ratio on the quality of banana preserves. Ciência Tecnologia Alimentos, V.32, n 2, p. 267-273, 2012.

SOLER, M. P.; FADINI, A. L.; HILST, M. A. S.; OKADA, C. E. Frutas, compotas, doce em massa, geleias e frutas cristalizadas para micro e pequena empresa. Campinas, SP: Instituto de Tecnologia de Alimentos (ITAL), (Manual Técnico). 1995. 
SOUZA, S. M. F. C.; LIMA, K. C.; MIRANDA, H. F.; CAVALCANTI, F. I. D. Utilização da informação nutricional de rótulos por consumidores de Natal, Brasil. Revista Panamericana de Salud Pública, Washington, v. 29, n. 5, p. 337-343, 2011.

STONE, H.; SIDEL, J. L. Sensory evaluation practices. 3. ed. New York: Academic Press. 2004. $408 \mathrm{p}$.
VIEIRA, D. A. dos S.; CASTRO, M. A.; FISBERG, M., FISBERG, R. M. (2017). Qualidade nutricional dos padrões alimentares de crianças: existem diferenças dentro e fora da escola?. Jornal de Pediatria, 93(1), 47-57. 